\title{
Trajectory Generation Using A Modified Simple Shooting Method
}

\author{
Ashley Trent \\ Texas Tech University \\ Lubbock, TX 79409 \\ 806-742-2580 x258 \\ atrent@math.ttu.edu
}

\author{
Ram Venkataraman \\ Texas Tech University \\ Lubbock, TX 79409 \\ 806-742-2580 x239 \\ rvenkata@math.ttu.edu
}

\author{
David Doman \\ Air Force Research Laboratory \\ WPAFB, OH 45433 \\ 937-255-8451 \\ david.doman@wpafb.af.mil
}

\begin{abstract}
In this paper, we combine the theoretical result of Pontryagin's Minimum Principle and a new numerical method to obtain a fast algorithm for the trajectory design problem for a reusable launch vehicle. We work with the outer-loop equations for an aircraft and consider the angle-of-attack to be the input. We cast the trajectory design problem as an optimal control problem and use Pontryagin's Minimum Principle to obtain first order necessary conditions. These are in the form of a two-point boundary-value problem (TPBVP) and we solve them by means of the Modified Simple Shooting Method (MSSM). In recent work, the MSSM has been shown to be superior, both in speed and accuracy, for TPBVPs.
\end{abstract}

\section{TABLE OF CONTENTS}

1. Nomenclature

2. INTRODUCTION

3. Modified Simple Shooting

4. REENTRY Vehicle EXAMPle

5. NumERICAL RESUltS

6. CONCLUSiOnS

\section{NOMENCLATURE}

TPBVP Two point boundary value problem MSSM Modified Simple Shooting method

$x \quad$ State variables

$\lambda \quad$ Costate variables

$V \quad$ Velocity of the air vehicle with respect to an earth fixed frame

$\gamma \quad$ Flight path angle

$X \quad$ Downrange

$H \quad$ Altitude

$L \quad$ Lift

$D \quad$ Drag

$C_{D} \quad$ Drag coefficient

$C_{L} \quad$ Lift coefficient

$m \quad$ Mass of vehicle

$g \quad$ Acceleration due to gravity

$T \quad$ Time unit (90 s)

$\alpha \quad$ Angle of attack, the control parameter

$K_{1}, K_{2} \quad$ Parameters in cost function

$\varepsilon, \varepsilon_{1} \quad$ Tolerances in the MSSM algorithm

$\mathcal{H} \quad$ Hamiltonian function

\section{INTRODUCTION}

Recently several authors have studied the problem of trajectory redesign for hypersonic aircraft [7], [6]. The usual procedure for solving such problems is to set it up as a constrained optimal control problem. Lu and Schierman used direct methods to numerically solve the optimal control problems. Alternatively, one could use Pontryagin's Minimum Principle to obtain first-order necessary conditions for the optimal control problem and obtain a twopoint boundary-value problem (TPBVP).

Originally created to solve two-point boundary value problems (TPBVPs), the Modified Simple Shooting Method (MSSM) has been shown to be superior, both in speed and accuracy, to known methods for solving TPBVPs [2]. Since optimal control problems can be formulated with differential equations and boundary conditions, it seems feasible to propose that the MSSM could be used to solve problems in optimal control. Here, the original MSSM algorithm (given with detail in [2]) was altered and used in conjunction with Pontryagin's Minimum Principle in an attempt to solve an optimal control problem in trajectory generation.

In this paper, we consider the problem of trajectory redesign of the unpowered reentry phase for a hypersonic air vehicle. We consider outer loop equations governing the motion of the center of mass and consider the angle of attack to be the input variable. The lift and drag forces for the aircraft considered were obtained from a polynomial neural network approximation of experimental data. Effector deflections were chosen so that the aircraft underwent trimmed flight.

\section{Modified Simple Shooting}

The Modified Simple Shooting Method has been used to successfully solve TPBVPs [2]. Using Pontryagin's minimum principle [5], we can obtain first-order necessary conditions for an optimal control problem and apply an adapted version of the MSSM to solve the resulting TPBVP.

0-7803-8155-6/04/\$17.00 2004 IEEE 
Suppose we want to minimize the cost function

$$
J=\int_{t_{0}}^{t_{1}} l(x, u) d t
$$

subject to

$$
\begin{aligned}
& \dot{x}=f(x, u), \\
& x\left(t_{0}\right)=x_{0}, x\left(t_{f}\right)=x_{f} \text { fixed, } \\
& u(\cdot) \in \Omega(t) .
\end{aligned}
$$

The following equation can be formed from (1) and (2),

$$
\dot{\lambda}(t)=-f_{x}^{\prime} \lambda(t)-l_{x}^{\prime},
$$

where $\lambda$ are called the costates. The pre-Hamiltonian is defined by

$$
\mathcal{H}(x, u, \lambda)=\lambda^{\prime}(t) f(x, u)+l(x, u) .
$$

Let $x^{*}(t), u^{*}(t)$ be the optimal solution. Then Pontryagin's Minimum Principle provides the Hamiltonian [4],[5]:

$$
\mathcal{H}\left(x^{*}(t), u^{*}(t), \lambda(t)\right)=\inf _{v \in \Omega(t)} \mathcal{H}\left(x^{*}(t), v, \lambda(t)\right)
$$

for all $t \in\left[t_{0}, t_{f}\right]$. The above equation can also be stated as:

$$
u^{*}(t)=\underset{v \in \Omega(t)}{\operatorname{argmin}} \mathcal{H}(x, v, \lambda) .
$$

Equations (2),(3),(5), and (7) constitute a TPBVP. To solve the problem, one needs to compute the value of $\lambda\left(t_{0}\right)$. Once this is done, the functions $x(t), u(t)$, are known for all $t \in\left[t_{0}, t_{f}\right]$. We propose to use the MSSM [2] to solve this TPBVP.

Two assumptions are needed for convergence of the algorithm [2].

Assumption 2.1: There exists an unique solution to the optimal control problem (2-5).

Assumption 2.2: Let $\hat{\lambda}\left(t_{0}\right)$ be the initial condition of the costates that results in the solution $(x(t), \lambda(t)$ to $(2-5)$. For every initial condition $\hat{\lambda}\left(t_{0}\right)$ within a small neighborhood of $\lambda^{*}\left(t_{0}\right)$ there is a unique solution defined on $\left[t_{0}, t_{f}\right]$. The solution is continuously differentiable with respect to changes in the initial conditions of the costates.

The algorithm proceeds as follows: Given a distance metric $d(\cdot, \cdot)$ on $\mathbb{R}^{n}$,

- Choose $\lambda(0)$ and reference path $\phi(t)$ such that $\phi\left(t_{0}\right)=$ $x_{0}, \phi\left(t_{f}\right)=x_{f}$, and $\phi(t)$ is sufficiently close to the optimal solution. Pick sufficiently small numbers $\varepsilon_{1}$ and $\varepsilon$ such that $\varepsilon_{1}>\varepsilon$.

- At time-step $k$ :

- Compute $u(k)$ using $x(k), \lambda(k)$ and (8).

- Integrate (2), (5), and compute $x(k+1), \lambda(k+1)$.
- If $d(\phi(k+1), x(k+1))>\varepsilon_{1}$, then apply the modified Newton's method and correct $\lambda(0)$ so that $d(\phi(k+$ $1), x(k+1))<\varepsilon$. Let $k=k+1$.

- Else (we have reached the final time), apply the modified Newton's method and correct $\lambda(0)$ so that $d\left(x_{f}, x\left(t_{f}\right)\right)<\varepsilon$.

- Stop.

Notice in this adapted version of the algorithm, initial values are guessed and corrected for the costates $\lambda$. If $\varepsilon_{1}$ is too small, the numerical method might have difficulties in convergence. From calculus of variations it is known that changes in the costates will affect the outcome of the states. Optimization of the control variable is performed at each step of the algorithm.

\section{REENTRY VEHICLE EXAMPLE}

The following equations are sometimes used to model a reentry vehicle during approach and landing. Here, only the equations of longitudinal motion are considered, describing the motion of the center of mass of the vehicle subject to external forces. This set of equations applies primarily to the performance of the vehicle, while the sets of moment and elastic equations are less influential [1].

For $t \in\left[t_{0}, t_{f}\right]$, the equations of motion are given as

$$
\begin{aligned}
\dot{V} & =\left(\frac{-D}{m}-g \sin \gamma\right) \\
\dot{\gamma} & =\left(\frac{L}{m V}-\frac{g}{V} \cos \gamma\right) \\
\dot{X} & =V \cos \gamma \\
\dot{H} & =V \sin \gamma,
\end{aligned}
$$

where $V$ is the velocity, $\gamma$ is the flight path angle, $X$ is the downrange position, and $H$ is the altitude. Lift and drag are given by

$$
\begin{aligned}
L & =\bar{q} S C_{L}(\alpha) \\
D & =\bar{q} S C_{D}(\alpha) \\
\bar{q} & =\frac{1}{2} \rho(H) V^{2},
\end{aligned}
$$

with the value $\rho(H)$ as the standard atmospheric density dependent upon the altitude $H$. The values $C_{D}$ and $C_{L}$ are the coefficients for drag and lift, respectively and dependent upon the angle-of-attack, $\alpha$ (see Section 4 for details).

Constraints on the control variable and states at any given time $t$ are

$$
\begin{aligned}
& 0^{\circ}<\alpha<15^{\circ} \\
& H(t)>2177 \mathrm{ft} \\
& V(t)>290 \mathrm{ft} / \mathrm{sec}
\end{aligned}
$$


The cost function, used to minimize the pitching moment $M_{0}$ of the wing body, is

$$
J=\int_{0}^{t}\left[K_{1}\left(\alpha-\alpha_{\text {des }}\right)^{2}+K_{2} \dot{\gamma}^{2}\right] d t
$$

where $\alpha_{d e s}=5^{\circ}$, the angle at which the minimum wingbody static pitching moment occurs for the vehicle.

For this example, the initial conditions given for $t=0$ seconds are

$$
\begin{aligned}
V(0) & =466 \mathrm{ft} / \mathrm{sec} \\
\gamma(0) & =-29^{\circ} \\
X(0) & =-15754 \mathrm{ft} \\
H(0) & =10066 \mathrm{ft} .
\end{aligned}
$$

The final condition is $H\left(t_{f}\right)=2274 \mathrm{ft}$, with constraints on the final values for $V, X$ and $\dot{H}$ as follows.

$$
\begin{aligned}
& V\left(t_{f}\right)>342 \\
& 0 \mathrm{ft}<X\left(t_{f}\right)<6000 \mathrm{ft} \\
& \dot{H}\left(t_{f}\right)>-39 \mathrm{ft} / \mathrm{sec}
\end{aligned}
$$

Using the constraint on $\dot{H}$, one may obtain a final constraint $\gamma\left(t_{f}\right)>-6.5^{\circ}$.

\section{NumERICAL RESUlTS}

Before applying the algorithm, the equations of motion (9-12) were scaled so that the solutions were of the same magnitude. Scaling constants were $g=32.2 \mathrm{ft} / \mathrm{s}$ and $T=90 \mathrm{~s}$. Accelerations were scaled by $g$, velocities were scaled by $g T$, and positions were scaled by $g T^{2}$.

Each of the sources for lift and drag coefficients were obtained from a polynomial neural network (PNN) model of aerodynamics data containing the information for lift and drag on a solid body with aerodynamic forces acting on it. A polynomial fit to this PNN was used as the first source of lift and drag. The second source was created using the PNN in conjunction with an aircraft model to develop a table of aerodynamics data on an aircraft undergoing trimmed flight.

\section{Normal Flight Condition}

For the first attempt at solving this optimal control problem, the lift and drag coefficients were approximated with polynomials of the angle of attack $\alpha$ [3].

$$
\begin{gathered}
C_{D}=0.0008 \alpha^{2}-0.0015 \alpha+0.1269 \\
C_{L}=0.0737 \alpha-0.0460
\end{gathered}
$$

The set of polynomials was the simplest form of the lift and drag coefficients to implement. Thus, it was used first to obtain an initial solution and to compare with future results.
Optimization was accomplished using a necessary condition for the minimum of the Hamiltonian: if $\alpha$ is the optimal control for (2), then $\mathcal{H}_{\alpha}(x, \alpha, \lambda, t)=0$. $\mathcal{H}_{\alpha}$ was calculated and set equal to zero to get a formula for obtaining the optimal control $\alpha$. This routine is justified in that, for this cost function (1), $\mathcal{H}_{\alpha \alpha}>0$, thus the extremal $\alpha$ is in fact a minimum.

The algorithm obtained a solution in 2.18 seconds with four iterations of the loop (see Figure 1). Corrections to $\lambda(0)$ occurred until the solution at time $k$ fell within $\varepsilon_{1}=0.15$ of the reference trajectory. Integration was halted when the solution at the final time was within $\varepsilon=0.01$ of $x_{1}$. For this case, the control profile stays within the given constraints for $\alpha$ (16) (see Figure 2).

\section{Normal Flight Condition with Trimmed Flight}

Using the MSSM with the tables reflecting lift and drag coefficients from trimmed flight, results were obtained for a normal flight situation.

TOMLAB's ucsolve function was used for the solution of (7). Convergence of the MSSM occurred after 331 seconds. The intermediate tolerance $\varepsilon_{1}$ was set at 0.1 , with the final tolerance $\varepsilon$ at 0.25 . The control profile remains within bounds (see Figure 4).

\section{A Failure Case with trimmed flight}

Since the adapted version of the MSSM seemed to work sufficiently well in solving a "normal" flight case, it was used to attempt a solution for the case where one control surface had failed to respond to commands. That is, one of the four control surfaces on the aircraft's body has failed and is locked in position. Failures of the aircraft control surfaces can cause increases in drag, decreases in lift, and overall instability of the aircraft. The pilot or piloting program then becomes incapable of deflecting the failed control surface to maintain stable flight.

For this particular situation, the aircraft's left flap has failed at $30^{\circ}$ down. This was modeled by the lift and drag coefficients, which were obtained from a table of aerodynamics data formed from the PNN with an aircraft model whose left flap was deflected at $30^{\circ}$ down. The same implementation for the MSSM was used as in Section 4. The final time was changed to $t_{f}=81$ seconds for feasibility. The tolerances were relaxed also, with $\varepsilon=1$ and $\varepsilon_{1}=0.2$. The algorithm took 2576 seconds to converge, and constraints were violated for $V$ and $\dot{H}$ (24) (see Figure 5). However, the control profile stays within the given constraints (Figure 6).

\section{CONCLUSIONS}

The MSSM was shown to be successful in solving the sensitive optimal control problem of trajectory design. The 

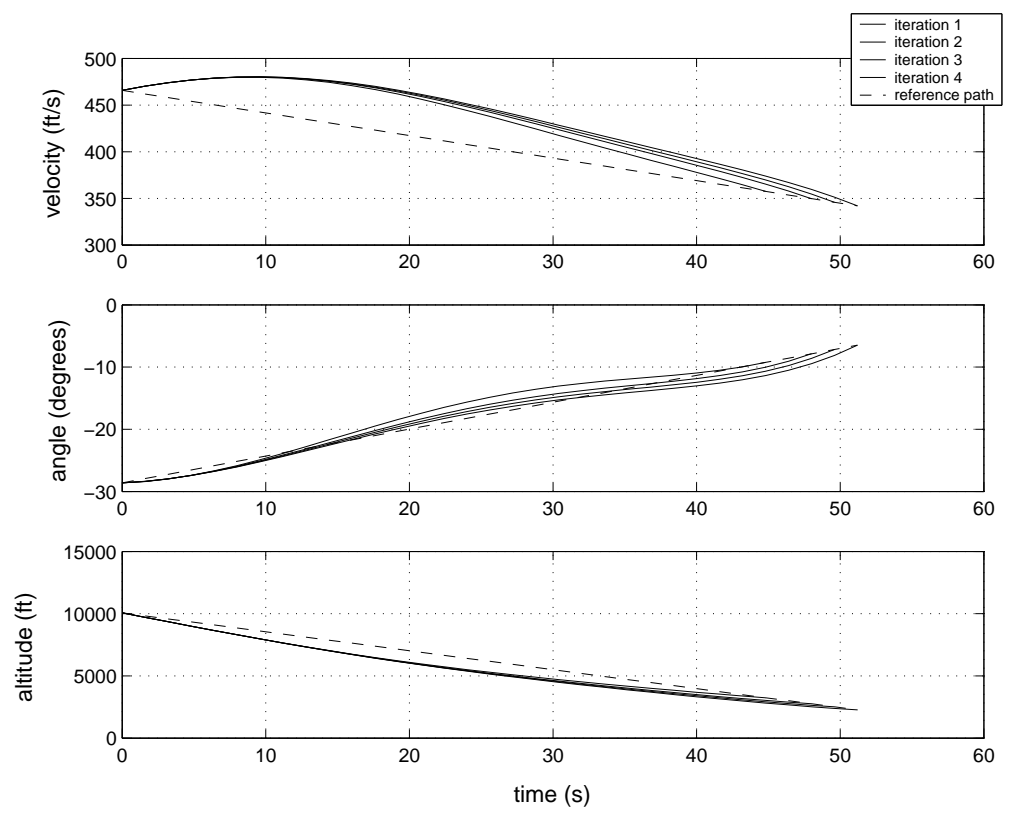

Figure 1. States resulting from MSSM using polynomials for drag and lift coefficients

unfailed case resulted in a reasonable solution and control profile. It may be that the failed case is an infeasible problem to solve without allowing for some increased angular accelerations. More investigation is needed before substantial conclusions can be made on the failure situation.

Current research involves reformulating the problem to a time-invariant set of equations using the monotonically increasing function $X(t)$. Future work includes attempting other cost functions in place of (1). Other failures are to be explored using this algorithm. Continued research on this and better implementations of the algorithm will provide improved solutions for the trajectory design optimal control problem.

\section{ACKNOWLEDGEMENT}

We would like to acknowledge Dr. F. Fahroo and Dr. M. Bolender for helping us with this research. A. Trent was supported by an AFRL, Air Vehicles Directorate Summer Research Program Fellowhip, and R. Venkataraman was supported by a NRC Summer Faculty Fellowship during Summer 2003, when this research was performed.

\section{REFERENCES}

[1] Bernard Etkin. Dynamics of Flight - Stability and Control. John Wiley and Sons, Inc., Toronto, second edition, 1982.

[2] Raymond Holsapple, Ram Venkataraman, and David Doman. A new, fast numerical method for solving two-point boundary value problems. Journal of Guidance, Control and Dynamics, 2003. To appear.

[3] Scott Josselyn and I. Michael Ross. Rapid verification method for the trajectory optimization of reentry vehicles. Journal of Guidance, Control and Dynamics, 26(3):505508, 2002.

[4] David G. Luenberger. Optimization by Vector Space Methods. John Wiley and Sons, Inc., New York, 1969.

[5] L. S. Pontryagin, V. G. Boltyanskii, R. V. Gamkrelidze, and E. F. Mishchenko. The Mathematical Theory of Optimal Processes. John Wiley and Sons, Inc., New York, 1962. Authorized translation from the Russian by K. N. Trirogoff.

[6] J. D. Schierman, J. R. Hull, and D. G. Ward. Online trajectory command reshaping for reusable launch vehicles. In Proceedings of the AIAA Guidance, Navigation and Control Conference, number AIAA-2003-5349, 2003.

[7] Zuojun Shen and Ping Lu. On-board generation of threedimensional constrained entry trajectories. In Proceedings of the AIAA Guidance, Navigation and Control Conference, number AIAA-2002-4455, 2002. 


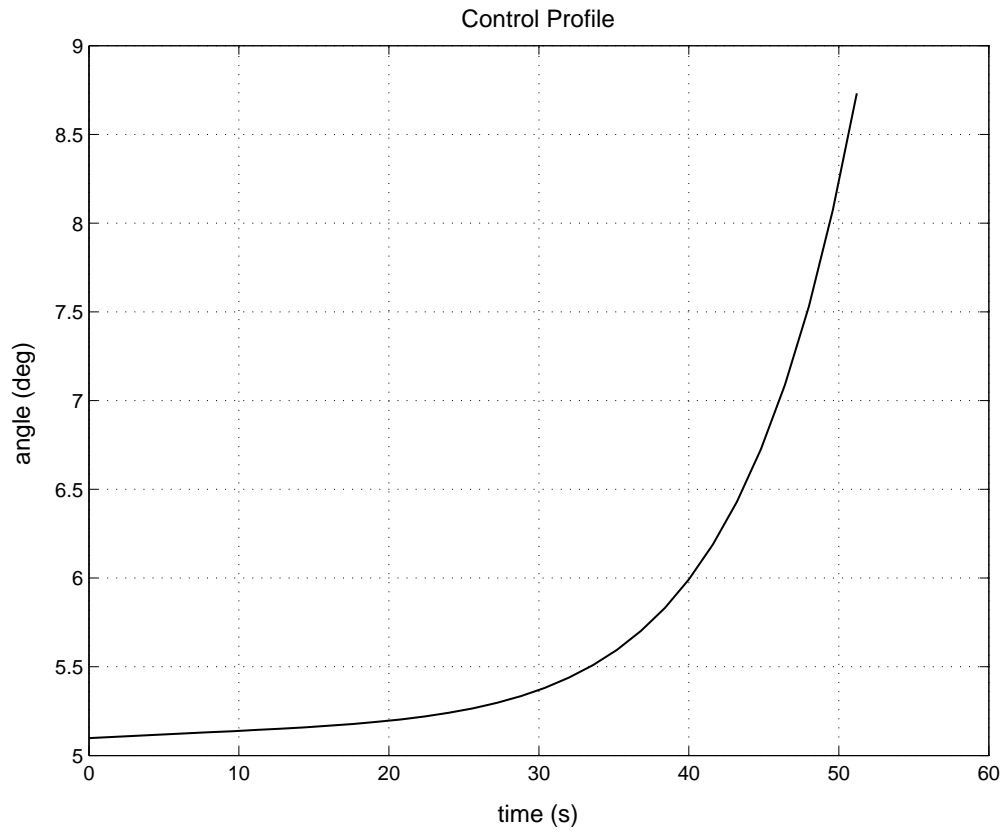

Figure 2. Control profile from MSSM using polynomials for drag and lift coefficients

Ashley Trent is a graduate student at Texas Tech University, currently working on a master's degree in applied mathematics. She has a B.S. in mathematics from Elon University.

Ram Venkataraman currently holds an Assistant Professor position in the Department of Mathematics and Statistics at Texas Tech University. He has a Ph.D. in Electrical Engineering from the University of Maryland at College Park. His research interests include differential geometry and functional analysis, and their applications to system, control theory and smart structures.

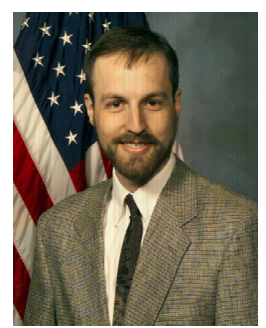

../David Doman is a senior Aerospace Engineer at the Control Theory and Optimization Branch, Control Sciences Division, Air Force Research Laboratory, WPAFB, OH. He is the technical lead for the hypersonics program at AFRL. He has a Ph.D. in Aerospace Engineering from Virginia Tech, and is a senior mem- 

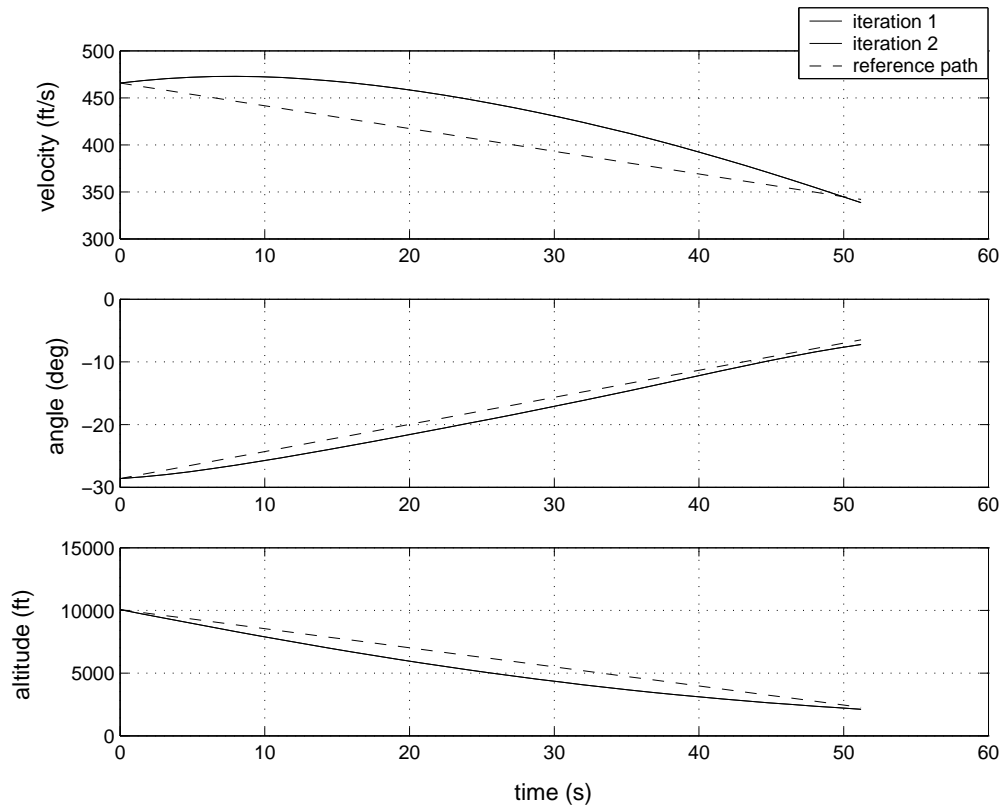

Figure 3. States resulting from MSSM using tables for trimmed flight

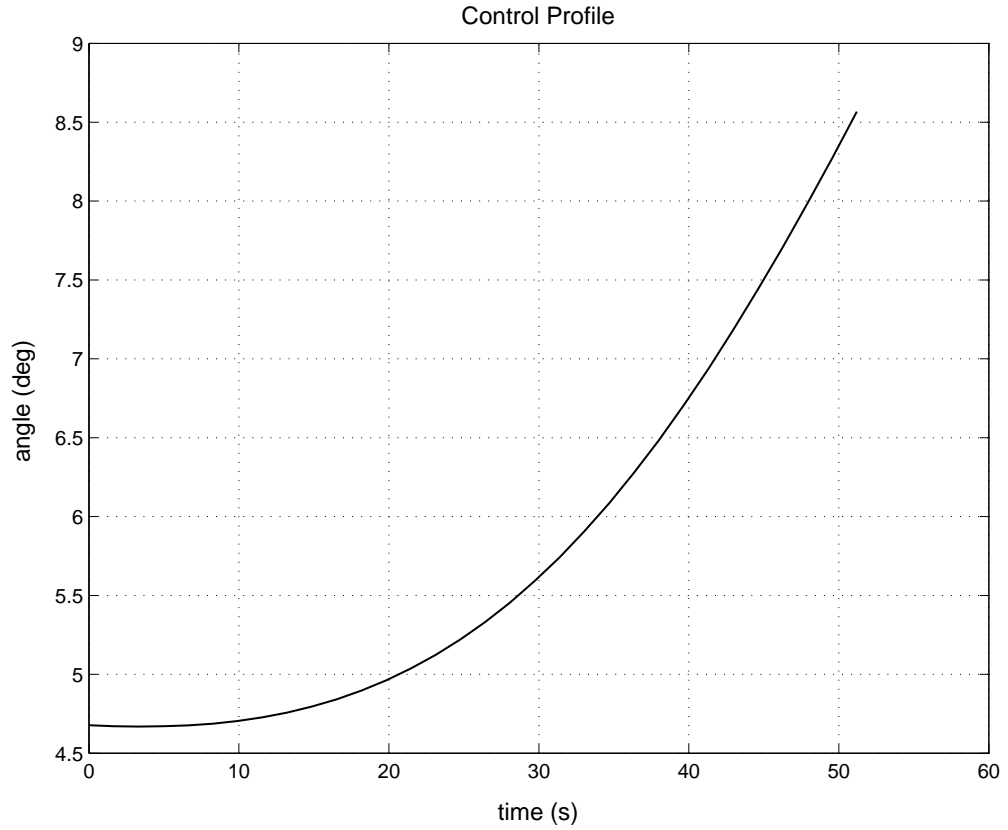

Figure 4. Control profile from MSSM using tables for trimmed flight 

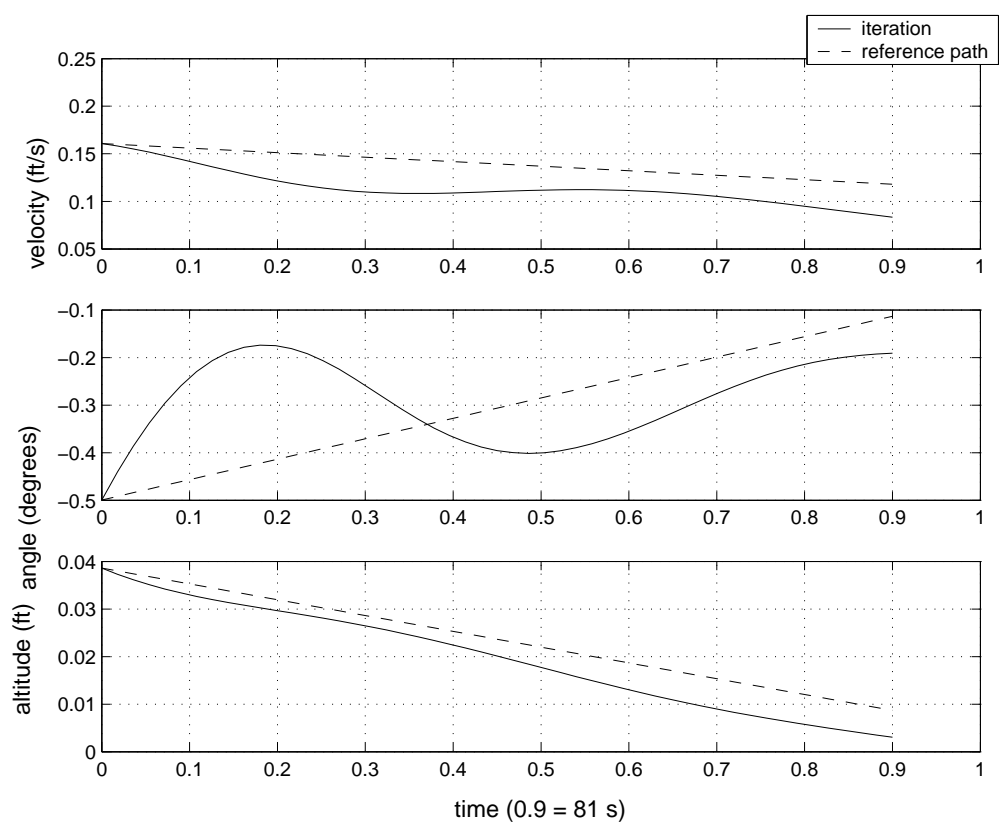

Figure 5. States resulting from MSSM using tables for trimmed flight

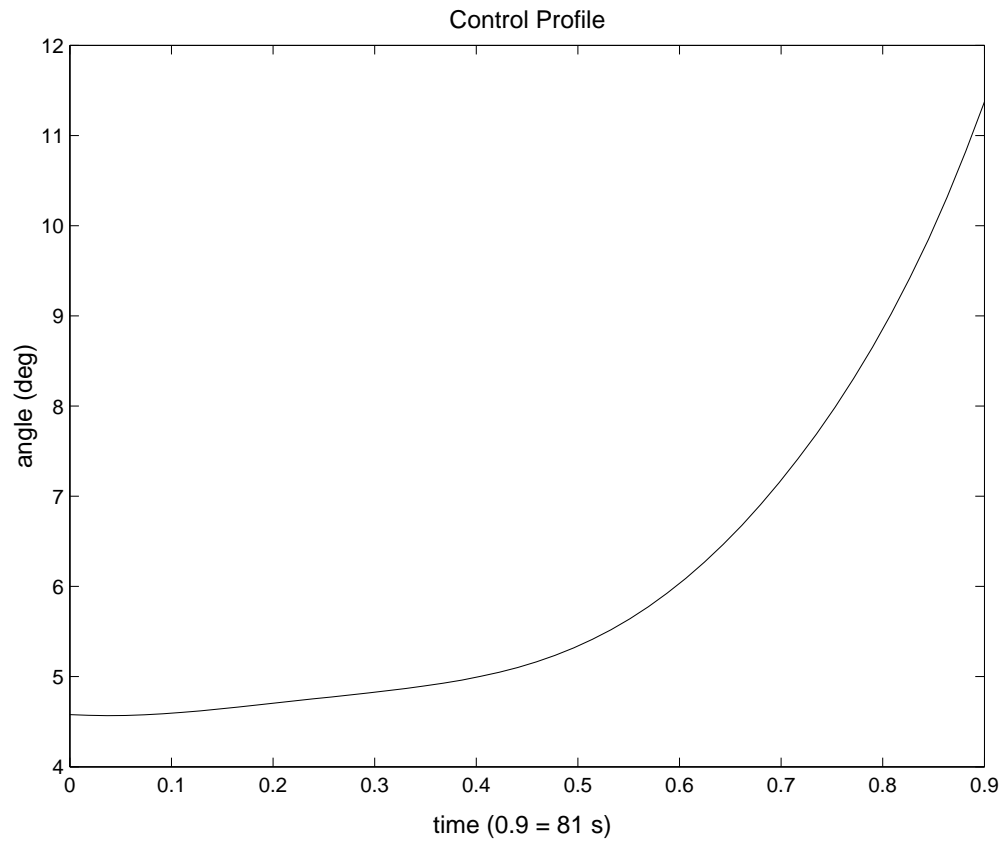

Figure 6. Control profile from MSSM using tables for trimmed flight 